\title{
Design of the propelling nozzles for the launchers and satellites
}

\author{
Rabah Haoui* \\ University of sciences and technology houari boumediene
}

\begin{abstract}
The aim of this work is to determine supersonic nozzle profiles that are used in propulsion, for launchers or embarked with satellites. This design has the role of firstly, providing important propulsion, i.e. with uniform and parallel flow at exit; and secondly, to find short length profiles, without modification of the flow in the nozzle. The first elaborate program is used to determine the profile of the divergent, by using the characteristics method for an axisymmetric flow. The second program is conceived by using the finite volume method, to determine and test the profile found connected to a convergent.
\end{abstract}

Key words: Characteristic method, nozzles, supersonic flow, propellers

\section{Introduction}

In aeronautics, as in aerospace, conduits constitute the essential body in propulsion. The relaxation of gases in the conduit after the combustion chamber, where there is great pressure, to the exit where there is low pressure, generally atmospheric pressure or the vacuum of space, provides a force of propulsion that moves the vehicle. To achieve this objective, it should be taken care that the flow in the nozzle is without the presence of a shock wave, and that it is uniform and parallel at the exit. The profile slope of the divergent starts from the throat with a zero value; then it increases, according to an arc of a circle, up to a maximum value, which is a function of the exit Mach number; and then it decreases gradually to the exit, or it becomes zero (Fig. 1).

Expansion waves are emitted by the first part, the arc of the circle, and are propagated in the flow, while entering into

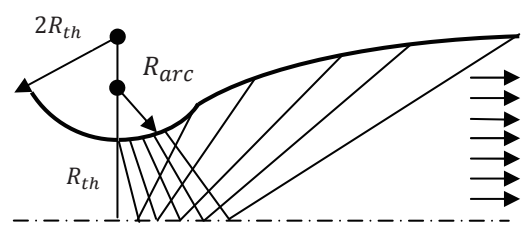

Fig. 1. Characteristic lines in the divergent interaction with those that are emitted by the lower wall. In the second part of the divergent, which is the required part, the waves are eliminated, since the slope of the wall decreases in such a way that the flow becomes uniform and parallel at the exit. Two important questions arise, which are the radius of the arc of circle $R_{\text {arc }}$ and its maximum slope. Several executions of the computational code will enable us to obtain the two necessary values to achieve the objective.

\section{Mathematic Formulation}

The equations of the method of the characteristics treating an axisymmetric compressible flow are given by two differential equations connecting the velocity $V$, the Mach number $M$, and the angle $\theta$ (Fig. 2), (Shapiro [1]). From each point, two line characteristics $\xi$ and $\eta$ are propagated, forming an angle $\alpha$ with the vector speed $V$, which itself forms an angle $\theta$ with the x-axis.

$$
\begin{aligned}
& u=V \cos \theta ; v=V \sin \theta ; \sin \alpha=\frac{1}{M} \\
& \frac{d y}{d x}=\tan (\theta \mp \alpha) \\
& \frac{1}{V}\left(\frac{d v}{d \theta}\right)=\mp \tan \alpha+\frac{\sin \alpha \cdot \tan \alpha \cdot \sin \theta}{\sin (\theta \mp \alpha)} \frac{1}{r}\left(\frac{d r}{d \theta}\right)
\end{aligned}
$$

This is an Open Access article distributed under the terms of the Creative Commons Attribution Non-Commercial License (http://creativecommons.org/licenses/by$\mathrm{nc} / 3.0 /$ ) which permits unrestricted non-commercial use, distribution, and reproduction in any medium, provided the original work is properly cited. 
The procedure of calculation is to find the flow parameters at point 3 , the intersection of the two characteristics $\xi$ and $\eta$ resulting from the two other points 1 and 2 (Fig. 3).

From equation (2), we have:

$$
\begin{aligned}
& r_{3}-r_{1}=\left(x_{3}-x_{1}\right) \tan (\theta-\alpha) ; \eta=\text { const } \\
& r_{3}-r_{2}=\left(x_{3}-x_{2}\right) \tan (\theta+\alpha) ; \quad \xi=\text { const }
\end{aligned}
$$

Multiplying the equation (3) by $d \theta \cdot \cot \alpha$, we obtain the form

$$
d \theta \pm \frac{\cot \alpha}{V} d V \mp \frac{\sin \alpha \cdot \sin \theta}{\sin (\theta \mp \alpha)} \frac{d r}{r}=0
$$

Thus,

$$
\begin{aligned}
& \left(\theta_{3}-\theta_{1}\right)+Q_{1}\left(V_{3}-V_{1}\right)-\frac{G_{1}}{r_{1}}\left(r_{3}-r_{1}\right)=0 ; \eta=\text { const } \\
& \left(\theta_{3}-\theta_{2}\right)-Q_{2}\left(V_{3}-V_{2}\right)+\frac{G_{2}}{r_{2}}\left(r_{3}-r_{2}\right)=0 ; \xi=\text { const }
\end{aligned}
$$

where,

$$
\begin{aligned}
& Q=\frac{\cot \alpha}{V} \\
& F=\frac{\sin \alpha \cdot \sin \theta}{\sin (\theta+\alpha)}(\xi=\text { const }) \\
& G=\frac{\sin \alpha \cdot \sin \theta}{\sin (\theta-\alpha)}(\eta=\text { const })
\end{aligned}
$$

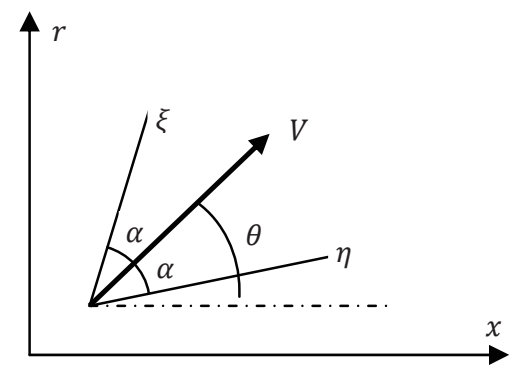

Fig. 2. Characteristics lines at a point

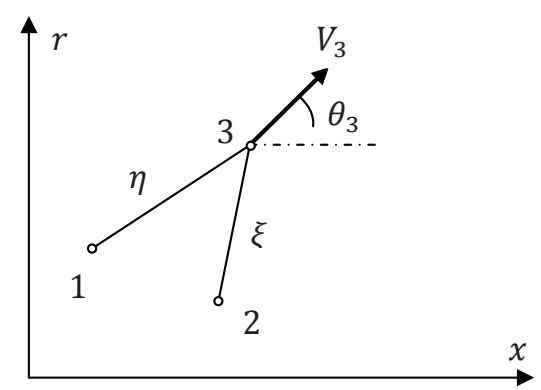

Fig. 3. Intersection of the two characteristics

\section{Calculation And Programming}

First, it is necessary to calculate the minimum radius of the arc of the circle. This arc must meet the Mach wave $\xi$ coming on the opposite side. The angle of inclination of this wave is a function of the throat Mach number $M_{t h}$ on the side of the wall, such as:

$$
\beta_{t h}=f\left(M_{t h}\right)=\operatorname{asin}\left(\frac{1}{M_{t h}}\right)
$$

The Mach number $M_{t h}$ is taken nearer to the unit. The problem consists here of finding the radius of the arc $R_{a r c}$ in such way that the intersection would take place at the point where the angle of the wall equals $\theta_{\max }$. In this case, one supposes that the Mach wave does not become deformed, but remains a straight line. It is easily shown that:

$$
\frac{R_{\text {arc }}}{R_{t h}}=\frac{2}{\sin \theta_{\text {max }} \cdot \tan \beta_{t h^{-1}}+\cos \theta_{\max }}
$$

Figure 4 shows the variation $\left(\frac{R_{a r c}}{R_{t h}}\right)_{\min }$, according to the maximum angle of the wall. It is the minimum value to have a progressive distribution of the expansion waves, resulting from the concave wall of the circle arc. The more the ratio is lower than the minimum, the more the expansion waves are concentrated at the beginning of the divergent follow-up of a longer conical wall.

To avoid an exaggerated concentration of the relaxation waves, it is necessary to increase the radius of the arc. With this intention, several executions of our programme were made, until the first wave eliminated on the convex part becomes closer to the last wave emitted by the concave part. In this case, there will be a good join between the arc, and the remainder of the divergent one. The same procedure must be done for different exit Mach number of the nozzle, i.e. for various angles $\theta_{\max }$. One thus obtains the graph of Figure 5, for the plane and axisymmetric nozzles.

In 2D and for axisymetric nozzles, the following approximate relation is proposed:

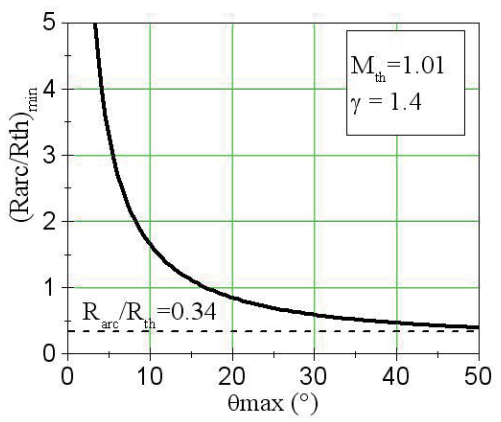

Fig. 4. Minimum radius of the arc versus angle 


$$
\left(\frac{R_{\text {arc }}}{R_{t h}}\right) \max =1.75+1.75 . \exp \left(-\frac{\theta_{\max }-12.03}{10.78}\right)
$$

In this relation, $\theta_{\max }$ is a function of the exit Mach number of the nozzle, which in 2D is given by the function of Prandtl Meyer $v(M)$, which is:

$$
v(M)=\sqrt{\left(\frac{\gamma+1}{\gamma-1}\right)} \operatorname{artan} \sqrt{\left(\frac{\gamma-1}{\gamma+1}\right)\left(M^{2}-1\right)}-\operatorname{artan} \sqrt{\left(M^{2}-1\right)}
$$

The maximum angle of inclination is $\theta_{\max }=\frac{v(M)}{2}$.

For the axisymmetric nozzle, $\theta_{\max }$ can be obtained numerically; it is function of $v(M)$, and the effect of axial symmetry. The following value is proposed (Fig. 6):

$$
\theta_{\max }=\frac{v(M)}{4 . \mathrm{k}}
$$

The coefficient $\mathrm{K}$ is a function of the nature of the gas, and the exit Mach number. For air, $k=0.82(\gamma=1.4$ and $M=3)$. For steam, it is the case of the engines cryotechnic, $\mathrm{k} \approx 0.8$, which varies little with the exit Mach number $M_{e}$ of the nozzle; that is to say:

$$
K=0.81-4.16710^{-4} * M_{e}+2.510^{-4} * M_{e}^{2}
$$

Concerning the variation of the section ratio of the nozzle according to the exit Mach number for the 2D case and axial

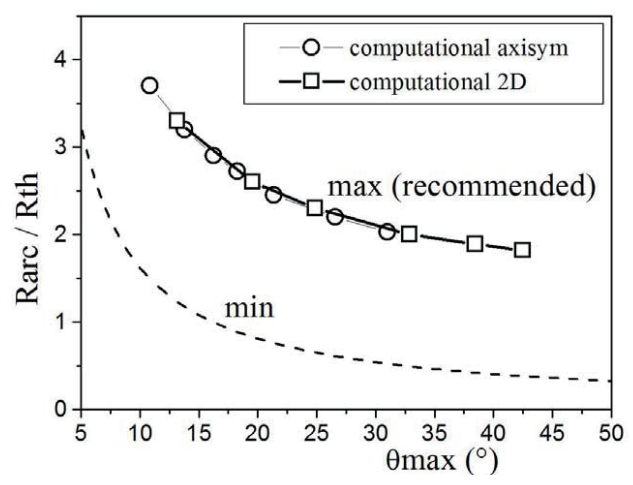

Fig. 5. Maximum radius of the arc versus angle

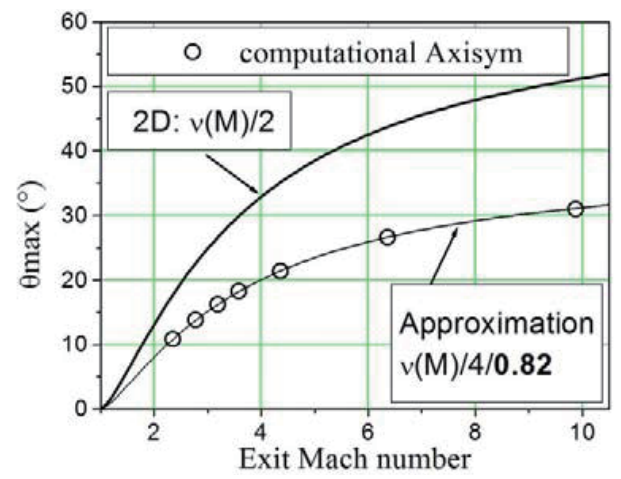

Fig. 6. Variation of the angle of inclination according to $M$ symmetry, it is the same one as in the monodimensional case, since the flow at the exit is uniform and parallel (Fig. 7).

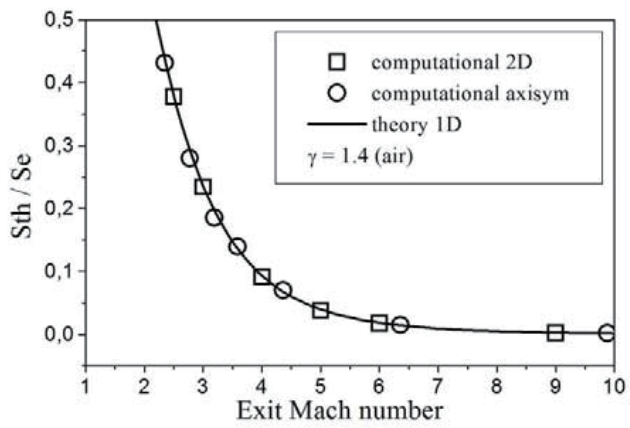

Fig. 7. Section ratio according to Me

\section{Results And Interpretation}

\subsection{Characteristic method}

The profile exactitude is a function of the size grid, i.e. the number of the Mach lines or the division used, $n$. In Figure 8 , we present several profiles, according to the number of divisions, $n=10,20,40$ and 60 . We note that for $n=60$, the profile already takes its final form, which does not change any more for $\mathrm{n}$ higher than 60 . The same observation applies to an axisymmetric profile (Fig. 9). Figure 10 shows the difference between 2D and axisymetric profiles giving the same Mach number at the exit, with a uniform and parallel flow. The section ratio is the same for both. Notice that the

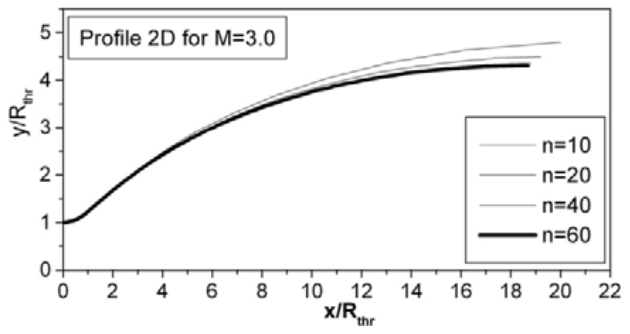

Fig. 8. 2D Profile, according to the grid size

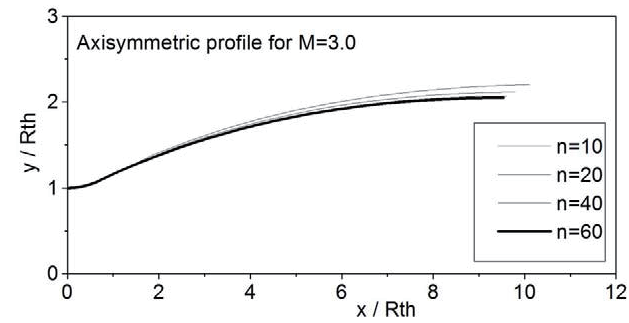

Fig. 9. Axisymetric profile, according to the grid size 
plane conduit is larger and longer than the axisymmetric nozzle.

To be sure that the calculation is well made, it is necessary to draw the characteristic lines in the calculation domain. Here, one presents only the axisymmetric profile. Fig. 11 shows the characteristic lines propagating after the wall in arc, then entering into interaction with those that come from the lower wall; all the lines are eliminated by the convex wall, before reaching the exit of the nozzle.

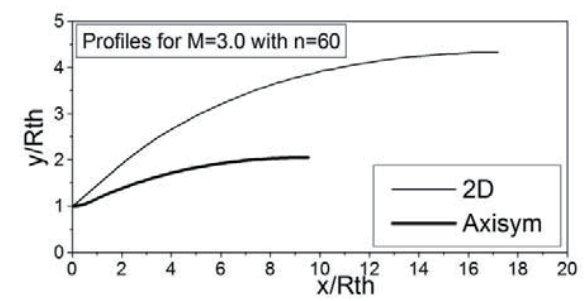

Fig. 10. Axisymmetric and 2D profiles for $M=3.0$

To see the axial symmetry of the flow, we can also draw the Mach contours in the divergent case, (Fig. 12).

Our objective is to determine the exact profile for a cryotechnic engine, whose characteristics are given in Table 1:

This type of nozzle, used in cryotechnic launchers, is called the Vulcan engine. Considering that the ideal nozzle obtained with the characteristics method is long, one uses a truncated ideal nozzle with $1 / 3$ of the divergent from the throat, because the important loss of kinetic energy is made at the beginning of the divergent. The advantage of the truncated nozzles is to gain much over the length; therefore, the weight is reduced, without much loss of thrust.
Table 1. Characteristics of engine nozzle

\begin{tabular}{|c|c|c|c|c|}
\hline Gas & $\gamma$ & $\mathrm{r}(\mathrm{J} / \mathrm{Kg} . \mathrm{K})$ & De $(\mathrm{m})$ & $\mathrm{L}(\mathrm{m})$ \\
\hline $\begin{array}{c}\text { Vapor } \\
\mathrm{H}_{2} \mathrm{O}\end{array}$ & 1.329 & 462 & $\mathbf{1 . 7 6}$ & $\mathbf{3 . 0}$ \\
\hline $\mathrm{qm}$ & $\mathrm{Ve}$ & $\mathrm{S} / \mathrm{Sth}$ & $\begin{array}{c}\mathrm{P} 0 \\
(\mathrm{bars})\end{array}$ & $\begin{array}{c}\mathrm{T} 0 \\
(\mathrm{~K})\end{array}$ \\
\hline $\mathrm{Kg} / \mathrm{s})$ & $(\mathrm{m} / \mathrm{s})$ & & 110 & 4000 \\
\hline 270 & 4000 & $\mathbf{4 5}$ & &
\end{tabular}

Several exit Mach numbers were tested in such way that at $1 / 3$ of the throat, the diameter was $D e=1.76 \mathrm{~m}$. The results that were obtained appear in Table 2.

The ideal nozzle is presented in Figure 13. The divergent profile of the truncated ideal nozzle is represented in Figure 14.

The truncated nozzle profile starts with an arc whose radius equals $0.2719 \mathrm{~m}$, until reaching a maximum slope of $27.068^{\circ}$, connected to a convex part, whose approximate form is exponential (Fig. 14).

We propose the following form:

$$
\begin{aligned}
& \frac{y}{R_{t h}}=y 1+A 1 \cdot \exp \left[-\frac{\left(\frac{x}{R t h}-x 1\right)}{t 1}\right] \\
& 0.943 \leq \frac{x}{R_{t h}} \leq 22.8 \quad ; \quad R_{t h}=0.13 m
\end{aligned}
$$

with:

$$
y_{1}=8.77, A_{1}=-7.90, x_{1}=0, t_{1}=17.158
$$

Figure 15 shows that the curve of approximation passes by

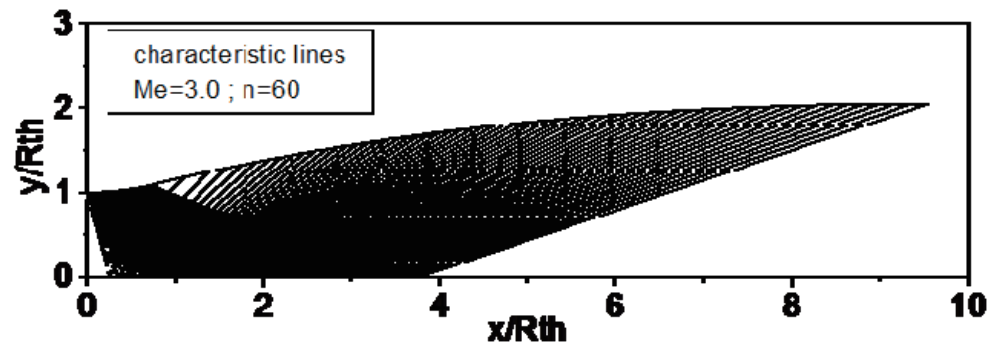

Fig. 11. Characteristic lines in the divergent of the nozzle

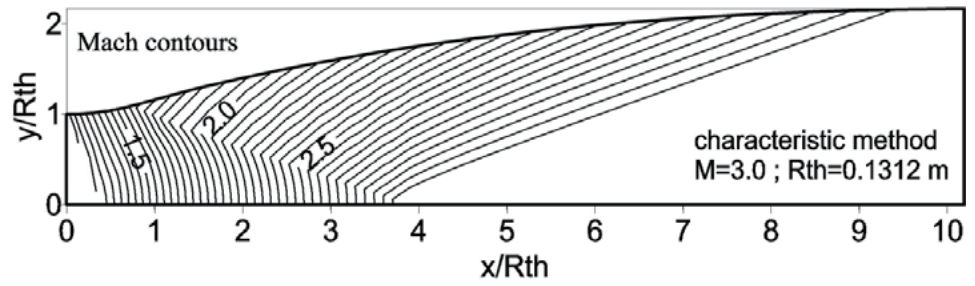

Fig. 12. Mach contours in the divergent of the nozzle 


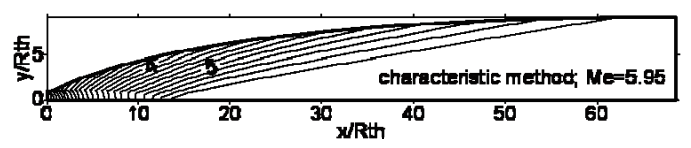

Fig. 13. Mach contours of the ideal nozzle, using the characteristic method

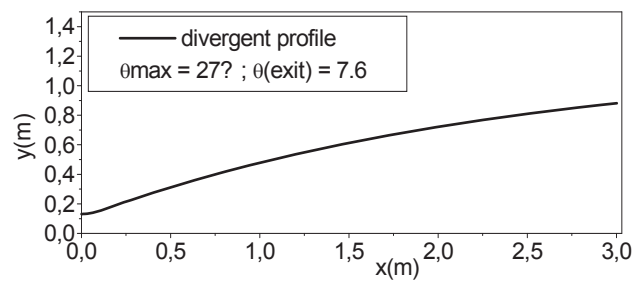

Fig. 14. Ideal truncated nozzle profile

Table 2. Characteristics of ideal and truncated nozzle

\begin{tabular}{|c|c|c|c|}
\hline \multicolumn{4}{|c|}{$\begin{array}{c}R_{t h}=.1312 m ; R_{\text {arc }}=0.2719, \gamma=1.329 \\
T_{0}=4000 K, \theta_{\max }=27.068^{\circ}\end{array}$} \\
\hline \multicolumn{4}{|c|}{ ideal nozzle (characteristic method) } \\
\hline$M$ & $V_{e}(\mathrm{~m} / \mathrm{s})$ & $L(\mathrm{~m})$ & $D_{e}(\mathrm{~m})$ \\
\hline 5.95 & 3570 & 9 & 2.426 \\
\hline$F_{p}(\mathrm{KN})$ & $q_{m}(\mathrm{Kg} / \mathrm{s})$ & $S / S_{t h}$ & $\theta_{\text {exit }}\left(^{\circ}\right)$ \\
\hline 1024.19 & 285.37 & 88 & 0 \\
\hline \multicolumn{4}{|c|}{ truncated ideal nozzle (CFD) } \\
\hline$M$ & $V_{e}(\mathrm{~m} / \mathrm{s})$ & $L(\mathrm{~m})$ & $D_{e}(\mathrm{~m})$ \\
\hline Axis $=5.62$ & Axis $=3542$ & \multirow{2}{*}{3.0} & \multirow{2}{*}{1.76} \\
\hline Wall $=4,78$ & Wall $=3440$ & & \\
\hline$F_{p}(\mathrm{KN})$ & $q_{m}(\mathrm{Kg} / \mathrm{s})$ & $S / S_{t h}$ & $\theta_{\text {exit }}\left(^{\circ}\right)$ \\
\hline 1019.74 & 285.12 & 45 & 7.63 \\
\hline
\end{tabular}

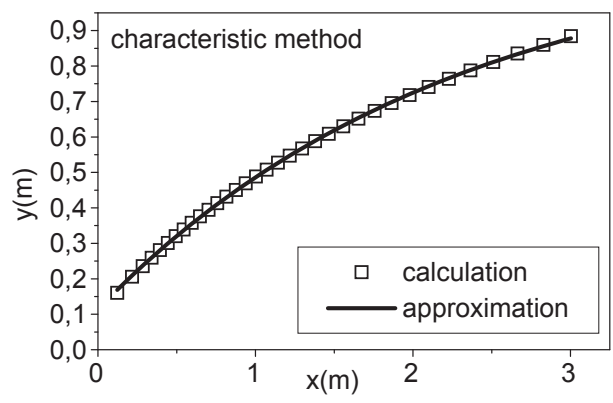

Fig. 15. Truncated nozzle profile approximation

all points constituting the profile of the truncated nozzle. The profile given by the preceding expression is reserved only for this nozzle, shown in Table 2, which corresponds well to the engine Vulcan 1 of the launcher ARIANE 5. It is the first family of profiles.

\subsection{CFD application}

We will test the profile found by the characteristics method with our computer code, by using the finite volume method of Van Leer [2], Haoui et al. [3], and Haoui [4], in order to determine the flow parameters in the nozzle, which starts by a convergence of conicity $45^{\circ}$, followed by an arc of a circle to the throat. The convergent is connected to the divergent profile found by the characteristics method. The grid used is represented in Figure 16.

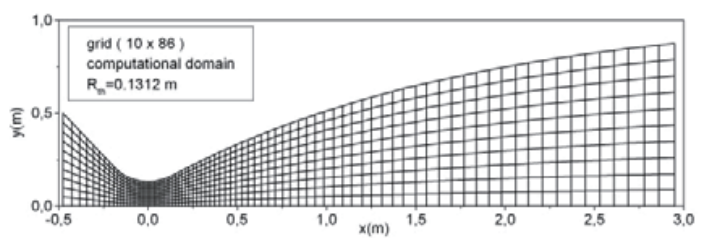

Fig. 16. Computational grid

The results are a function of grid size, number of iterations, and the CFL (Haoui et al. [5]). The interest in studying the flow by using the finite volume method is firstly, to compare with the characteristics method, and to see how the expansion waves are propagated in the nozzle, and the non-existence of a shock wave. Secondly, it is to be able to calculate the parameters at the exit of the truncated nozzle, especially the flow rate, and the thrust. Figure 17 shows that the Mach contours in all of the truncated nozzle almost form inclined lines in the right part of the divergent. It should be noted that the flow is modified a little, compared to the characteristic method.

The variation of the other parameters for the inviscid flow is represented in Figures 18, 19 and 20. It is noted that the axis Mach number is higher than that on the level of the wall, at the exit of the truncated nozzle. These are 5.62 and 4,78 , respectively, and the temperatures are $647 \mathrm{~K}$ and $844 \mathrm{~K}$, respectively. The pressures are of 0.055 bars and 0.192 bars, respectively. Notice that the pressure at the exit is lower than the atmospheric pressure at the time of launcher takeoff, which causes a compression of gas downstream from the nozzle, which will disappear after $15 \mathrm{~km}$ of altitude.

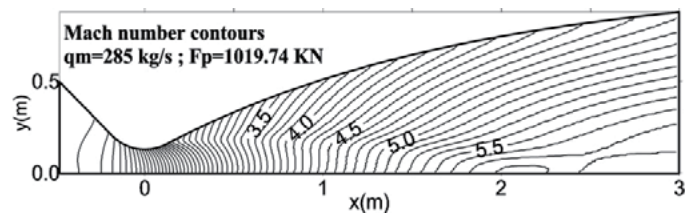

Fig. 17. Mach number contours in the nozzle 


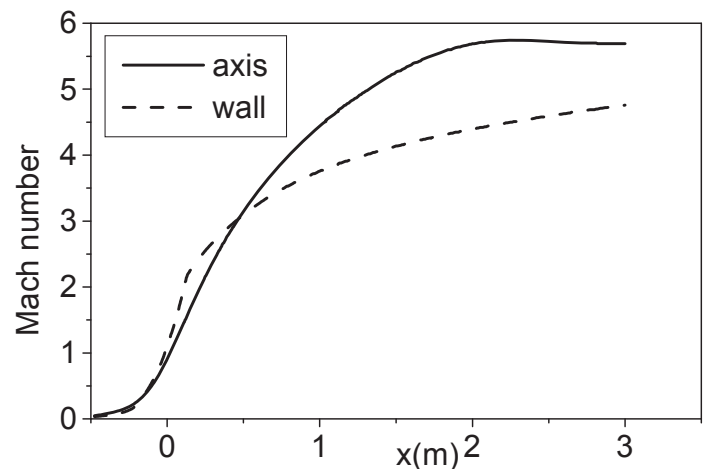

Fig. 18. Mach number evolution in the nozzle

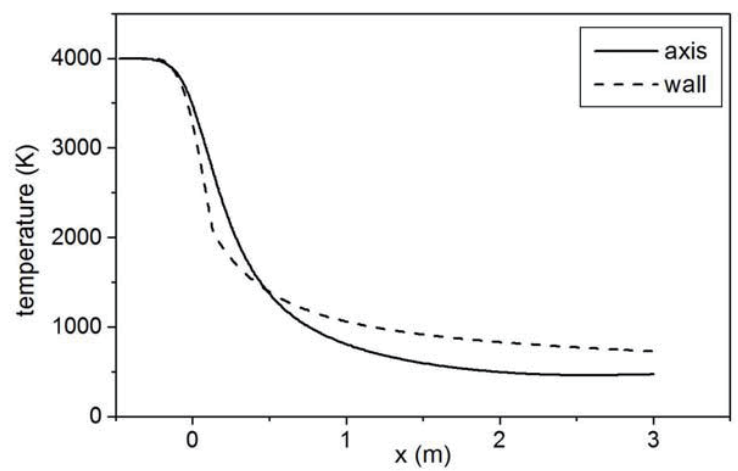

Fig. 19. Temperature evolution in the nozzle

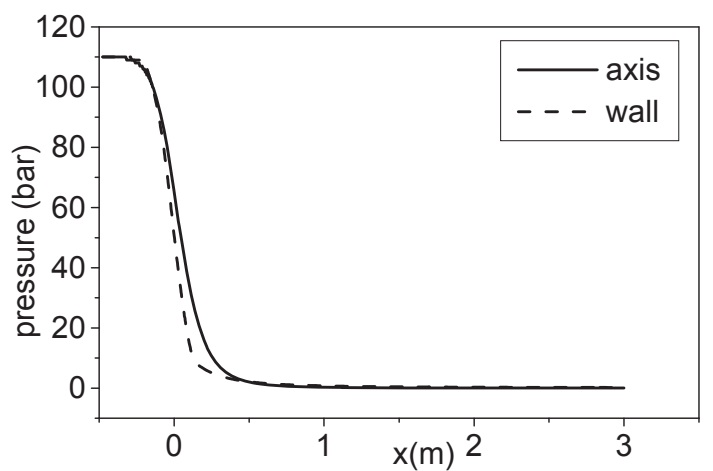

Fig. 20. Pressure evolution in the nozzle

\section{Conclusion}

We confirm that the profiles of the propelling nozzles are traced, firstly by using the method of characteristics in such way that the flow at exit is uniform and parallel. In this case, one obtains the ideal nozzle form, which is longer. Secondly, it is necessary to use the truncated nozzle deduced from the ideal nozzle, which must be tested by a computer code that takes account of the flow at the inlet of the convergent to the exit of the nozzle. Note of course that the results found in Table 2 are identical to those of the Vulcan 1 engine of the ARIANE 5 launcher.

\section{References}

[1] Shapiro, A.H., The Dynamics and Thermodynamics of Compressible fluid flow, The Ronald Press Company, New York. Volume II. Ch.17, 1954.

[2] Van Leer, B., "Flux Vector Splitting for the Euler Equations", Lecture Notes in Physics, Eighth International Conference on Numerical Methods in Fluid Dynamics, Vol.170, 1982, pp. 507-512.

[3] Haoui, R., Gahmousse, A. and Zeitoun, D., " Chemical and vibrational nonequilibrium flow in a hypersonic axisymmetric nozzle", International Journal of Thermal Sciences, Vol. 40, No.8, 2001, pp. 787-795.

DOI: S1290-0729(01)01265-0/FLA

[4] Haoui, R., "Finite volumes analysis of a supersonic non-equilibrium flow around the axisymmetric blunt body", International Journal of Aeronautical and space Sciences, Vol. 11, No.2, 2010, pp. 59-68. DOI:10.5139/IJASS.2010.33.1.059

[5] Haoui, R., Gahmousse, A. and Zeitoun, D., "Condition of convergence applied to an axisymmetric reactive flow", French Congress of Mechanics, Nice, France, 2003.

[6] Haoui, R., " Effect of Mesh Size on the Viscous Flow Parameters of an Axisymmetric Nozzle", International Journal of Aeronautical and space Sciences, Vol. 12, No.2, 2011, pp. 127-133. DOI:10.5139/IJASS.2011.12.2.93 